\title{
Regular surveillance for Li-fraumeni syndrome: advice, adherence and perceived benefits
}

\author{
C. R. M. Lammens $\cdot$ E. M. A. Bleiker - N. K. Aaronson - A. Wagner $\cdot$ R. H. Sijmons • \\ M. G. E. M. Ausems • A. H. J. T. Vriends • M. W. G. Ruijs • T. A. M. van Os • \\ L. Spruijt · E. B. Gómez García $\cdot$ A. Cats $\cdot$ T. Nagtegaal $\cdot$ S. Verhoef
}

Published online: 25 July 2010

(c) The Author(s) 2010. This article is published with open access at Springerlink.com

\begin{abstract}
Li Fraumeni Syndrome (LFS) is a hereditary cancer syndrome characterized by a high risk of developing various types of cancer from birth through late adulthood. Clinical benefits of surveillance for LFS are limited. The aim of this study is to investigate which advice for regular surveillance, if any, is given to high risk LFS individuals, adherence to that advice, and any psychological gain or burden derived from surveillance. Fifty-five high risk individuals (proven carriers and those at 50\% risk) from families with a p53 germline mutation were invited to participate, of whom $82 \%$ completed a self-report questionnaire assessing
\end{abstract}

E. M. A. Bleiker · M. W. G. Ruijs · S. Verhoef $(\bowtie)$

Family Cancer Clinic, The Netherlands Cancer Institute-Antoni

van Leeuwenhoek Hospital (NKI-AVL), Plesmanlaan 121,

1066 CX Amsterdam, The Netherlands

e-mail: s.verhoef@nki.nl

C. R. M. Lammens - E. M. A. Bleiker - N. K. Aaronson ·

T. Nagtegaal

Division of Psychosocial Research \& Epidemiology,

The Netherlands Cancer Institute-Antoni van Leeuwenhoek

Hospital (NKI-AVL), Amsterdam, The Netherlands

\section{A. Wagner}

Department of Clinical Genetics, Erasmus Medical Centre,

Rotterdam, The Netherlands

\section{R. H. Sijmons}

Department of Genetics, University Medical Center Groningen, University of Groningen, Groningen, The Netherlands

M. G. E. M. Ausems

Department of Medical Genetics, University Medical Centre

Utrecht, Utrecht, The Netherlands

\section{A. H. J. T. Vriends}

Department of Clinical Genetics, Leiden University Medical

Centre, Leiden, The Netherlands advice for regular surveillance, compliance, perceived benefits and barriers of screening and LFS-related distress (IES) and worries (CWS). In total, $71 \%$ of the high risk family members received advice to undergo regular surveillance for LFS. The majority (78\%) reported adherence with the recommended advice. All high risk women aged 25 or older reported having been advised to undergo annual breast cancer surveillance $(n=11)$, of whom $64 \%(n=7)$ in specific received advice to undergo a mammography. Seventy-eight percent of respondents indicated having received tailored surveillance advice based on family cancer

M. W. G. Ruijs

Department of Clinical Genetics, VU University Medical Centre, Amsterdam, The Netherlands

T. A. M. van Os

Department of Clinical Genetics, Amsterdam Medical Centre,

Amsterdam, The Netherlands

L. Spruijt

Department of Clinical Genetics, University Medical Centre Nijmegen, Nijmegen, The Netherlands

E. B. Gómez García

Department of Clinical Genetics, Academic Hospital Maastricht, Maastricht, The Netherlands

A. Cats

Division of Medical Oncology, The Netherlands Cancer Institute-Antoni van Leeuwenhoek Hospital (NKI-AVL), Amsterdam, The Netherlands 
history. The large majority of respondents believed in the value of surveillance to detect tumors at an early stage (90\%) and reported that it gave them a sense of control (84\%) and security (70\%). Despite its limited clinical benefits, the majority of high risk LFS family are advised to undergo, and are adherent to, and report psychological benefit from, regular surveillance programs.

Keywords Li-Fraumeni syndrome (LFS) - Surveillance · Compliance $\cdot$ Psychological barriers and benefits

\section{Introduction}

Li Fraumeni Syndrome (LFS) is a rare, autosomal dominant, inherited cancer syndrome characterized by a high risk of developing various types of cancer from birth through late adulthood. The only known cause of LFS is a germline mutation in the p53 gene [1]. Carriers of a p53 germline mutation have a life-time risk of $\sim 90 \%$ of developing a malignancy. The risk for women seems to be higher than for men, which partly can be explained by breast cancer occurrence [2,3]. Approximately $20 \%$ of the carriers develop cancer before the age of twenty [3], and may develop multiple primary cancers [4]. The most frequently occurring malignancies in LFS are pre-menopausal breast cancer, sarcomas, leukemia and brain tumors [5].

In cancer care, in general, the main aim of periodic surveillance (and of interventions that result from surveillance) is to prevent cancer or detect expression of the disease at the earliest possible stage in order to affect prognosis positively. In the more common forms of hereditary cancer, like hereditary breast and ovarian cancer (HBOC) and Lynch syndrome, periodic surveillance and risk reducing interventions have found to be beneficial in reducing mortality and morbidity in high risk family members [6-8]. In the case of LFS, however, debate is ongoing on which surveillance methods to offer and, in particular, what the medical gains of these methods are $[5,9,10]$. Given the wide range of tumor sites and age of onset, it is difficult to have effective periodic surveillance. Novel surveillance imaging techniques for LFS, such as full body MRI scan in thick slices and FDG-PET/CT scans, are currently being investigated [11]. However, at present, the only surveillance method that is thought to be effective is regular surveillance for breast cancer in women aged 20-25 or older. However, due to the possible radiation sensitivity associated with p53 mutations [12], there is considerable discussion about which breast surveillance method to use, mammography or breast MRI [5]. According to Varley et al., high risk individuals from families with a p53 mutation should avoid radiation whenever possible [13]. The diagnostic and preventive guidelines for LFS in the Netherlands state that, apart from regular breast cancer surveillance from the age of 20-25 years and onward, an annual anamnesis and physical examination could be considered [14].

To our knowledge, no study has investigated what type of advice for regular surveillance has actually been given to high risk LFS family members, or the extent to which such advice is followed. There is also a paucity of information on the perceived psychological barriers and benefits of regular surveillance for LFS. Given that LFS family members consider 'being able to plan surveillance as one of the most important reasons for undergoing genetic testing [15], we hypothesized that they would also experience psychological benefits from undergoing regular surveillance. Although the medical gains associated with regular surveillance remain the subject of debate, it may provide high risk family members with a sense of control over the disease [16].

The specific aims of this study were: (1) to evaluate what advice for regular surveillance is given to high risk LFS family members, (2) to assess adherence to the advice given, (3) to evaluate the self-reported reasons for (non)compliance with this advice; and (4) to investigate the perceived barriers and benefits of regular surveillance for LFS.

\section{Methods}

Study sample and procedures

As part of a larger, cross-sectional study performed in collaboration with all 9 family cancer clinics (FCC) in the Netherlands and the DNA-laboratory of the Netherlands Cancer Institute, 18 families with a p53 germline mutation were identified. Four of these families were ineligible because all registered adult family members were deceased at the time of the study. In two families, no high risk family members were identified. In total, 55 high risk family members (carriers and those at 50\% risk) from the remaining 12 families were invited to take part in the study. Family members were invited via their clinical geneticist. Those who could not be invited through the FCC, such as those at 50\% risk who had not received genetic counseling, were invited by a contact person within the family. Family contact persons were typically key figures within the family who assisted in drawing the family pedigree. Excluded from this part of the study were individuals younger than 16 years of age and those not aware that LFS runs in their family $(n=3)$. Eligible family members received an invitation letter and self-report questionnaire by mail. In case of non-response after 2 weeks, reminders per mail and telephone were used. Family members were recruited between August 2006 and February 2008. The 
study was approved by the institutional review boards of all participating hospitals.

\section{Measures}

\section{Sociodemographic and clinical variables}

Age, gender, marital status, education, offspring, personal history of cancer and DNA-status (carrier, 50\% at-risk, non-carrier) were obtained from the self-report questionnaire and the medical records.

\section{Advice for, and adherence to, regular surveillance}

A series of questions was posed to determine what methods, if any, for regular surveillance had been advised, and adherence to those recommendations. Compliers were defined as those who indicated that they had adhered to the advice given or more frequently than advised. Noncompliers were defined as those who do not undergo one or more of the advised surveillance methods and/or less frequently than had been advised. Where relevant, reasons for non- or under compliance were asked.

\section{Perceived benefits and barriers of screening}

Perceived benefits and barriers of regular surveillance for LFS were assessed with an 11-item scale adapted from previous work carried out by Champion [17], Kash et al. [18], and Madalinska et al. [19]. This included 5 "pro" statements and 6 "con" statements about regular surveillance. An example of a pro statement is: "Regular surveillance for LFS provides me with a feeling of control". An example of a con statement is: "Regular surveillance can cause unnecessary worry”. Response categories ranged on a five-point scale from 'strongly disagree' to 'strongly agree'. Sum scores for the pro and con subscales ranged from 5 to 25 , and 6 to 30, respectively. Reliability (Cronbachs' coefficient alpha) of the pro and con subscales was .83 and .45 , respectively.

\section{LFS-specific distress}

LFS-specific distress was measured with the 'intrusion' subscale of the Impact of Event Scale [20]. This 7-item questionnaire measures intrusive thoughts and feelings about LFS during the past 7 days. The IES-intrusion total score ranges between 0-35. A score of 9 or higher is considered to be clinically relevant [21], and additional psychosocial help may be indicated. The Dutch version of the IES has proven to be a valid and reliable instrument for assessing disease-specific distress [20]. Cronbach's alpha for the intrusion scale was 0.91 .

\section{LFS-related worries}

LFS-related worries were assessed with an 8-item questionnaire adapted from the Cancer Worry Scale (CWS) $[22,23]$. Scores range from 8 to 32 , with higher scores indicating more frequent worries about cancer. Cronbach's alpha in the present study was 0.89 .

\section{Statistical analysis}

Descriptive statistics were used to characterize the study sample, to describe the various recommended surveillance methods, to assess adherence with surveillance, and to investigate the perceived benefits and barriers of surveillance for LFS. Univariate analyses (analysis of variance, Student $t$ test, Fisher's exact test, chi square) were used to investigate possible differences between those who did and did not receive an advice for regular surveillance and between compliers and non-compliers in terms of sociodemographic, clinical and psychological variables. Because data on LFS-specific distress were positively skewed and leptokurtic, we log transformed the data in order to meet the assumptions for parametric testing. However, since the results using this method did not differ significantly from those based on parametric tests, we report here the results of the parametric test with the untransformed data only.

It was not possible to control for potential clustering effects (i.e., multiple family members from the same family) due to the fact that in four families only a single family member participated in the study.

All analyses were conducted using the Statistical Package for the Social Sciences (version 17.0).

\section{Results}

\section{Response}

In total, $45(82 \%)$ high risk family members (carriers and those at $50 \%$ risk) completed the questionnaire. No statistically significant differences were found between the 45 respondents and the 10 non-respondents for gender or age. The respondents stemmed from 9 LFS families.

Characteristics of the study sample

Sample sociodemographic characteristics are presented in Table 1. Twenty-seven individuals were carriers of a p53 mutation, of whom 10 had a cancer history. The remaining 18 individuals had a $50 \%$ risk of being a carrier of a p53 mutation. The majority of the participants was married or living together and had children. 
Table 1 Demographics study sample

\begin{tabular}{lllll}
\hline Characteristics & $\begin{array}{l}\text { Total } \\
(n=45) N(\%)\end{array}$ & $\begin{array}{l}\text { Compliers } \\
(n=25) N(\%)\end{array}$ & $\begin{array}{l}\text { Non-compliers } \\
(n=7) N(\%)\end{array}$ & $\begin{array}{l}\text { No advice } \\
(N=13) N(\%)\end{array}$ \\
\hline $\begin{array}{l}\text { Age (mean } \pm \text { SD (range)) } \\
\text { Gender (female) }\end{array}$ & $\begin{array}{l}40.6 \pm 15.2(17-75) \\
19(42 \%)\end{array}$ & $\begin{array}{l}44.8 \pm 14.4 \\
10(40 \%)\end{array}$ & $\begin{array}{l}39.7 \pm 13.7 \\
4(57 \%)\end{array}$ & $\begin{array}{l}32.2 \pm 15.1 \\
\text { Marital status }\end{array}$ \\
$\begin{array}{l}\text { Married/living together } \\
\text { Single }\end{array}$ & $31(69 \%)$ & $21(84 \%)$ & $6(86 \%)$ & $4(31 \%)$ \\
Education level & $14(31 \%)$ & $4(16 \%)$ & $1(14 \%)$ & $9(69 \%)$ \\
$\quad$ Low & $14(31 \%)$ & $9(36 \%)$ & $2(28 \%)$ & $3(23 \%)$ \\
$\quad \begin{array}{l}\text { Moderate } \\
\text { High }\end{array}$ & $18(40 \%)$ & $9(36 \%)$ & $3(43 \%)$ & $6(46 \%)$ \\
Children (yes) & $13(29 \%)$ & $7(28 \%)$ & $2(29 \%)$ & $4(31 \%)$ \\
Actual risk & $25(56 \%)$ & $17(68 \%)$ & $3(43 \%)$ & $5(39 \%)$ \\
Carrier & & & & \\
$50 \%$ at risk & $27(60 \%)$ & $20(80 \%)$ & $3(43 \%)$ & $4(31 \%)$ \\
Cancer history (yes) & $18(40 \%)$ & $5(20 \%)$ & $4(57 \%)$ & $9(69 \%)$ \\
\hline
\end{tabular}

Fig. 1 Flowchart advice and adherence to regular surveillance for LFS

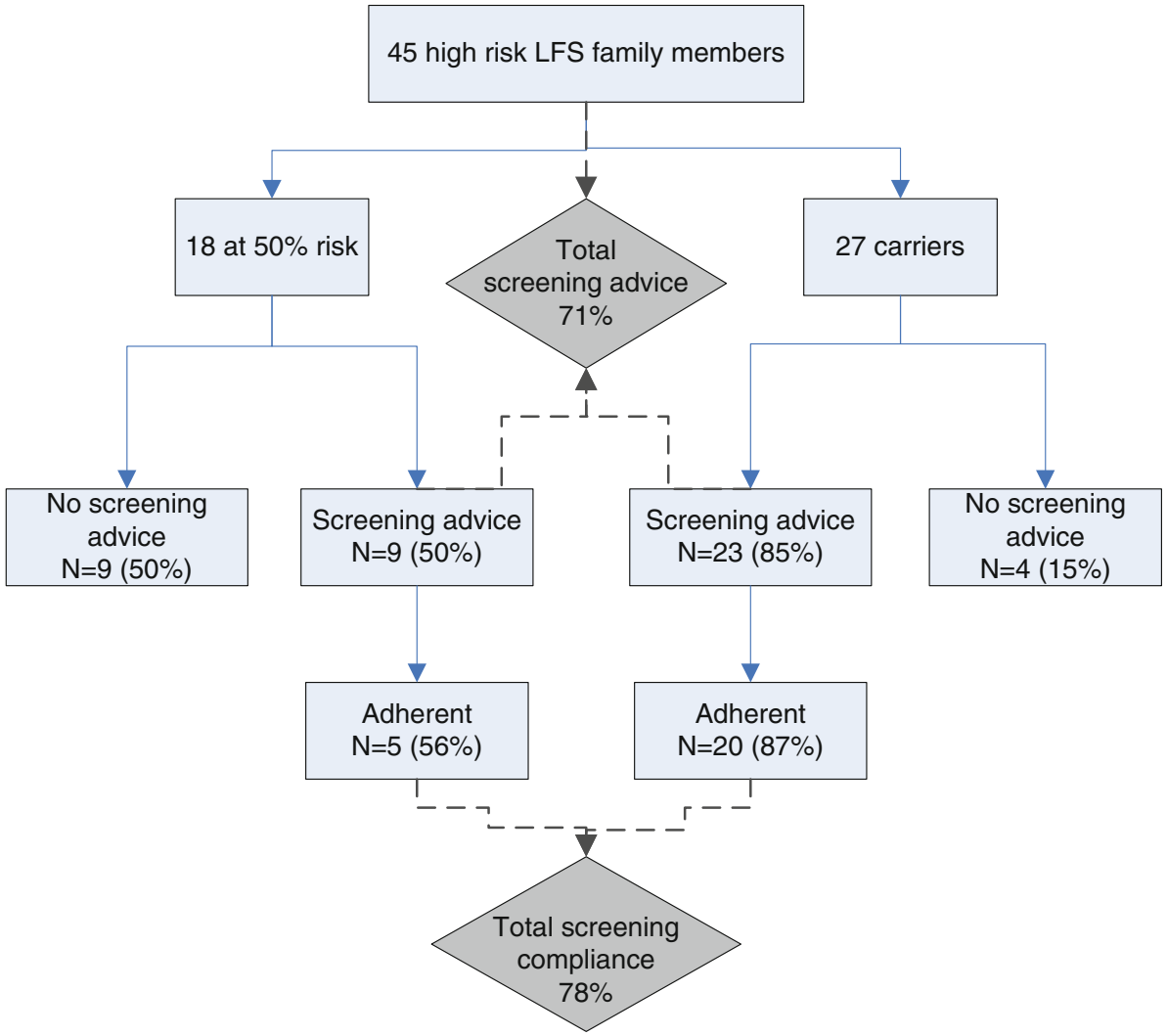

Surveillance advice

Of the total sample, 32 individuals (71\%) received advice to undergo regular surveillance for LFS-related conditions. Fifty percent $(n=9)$ of those at $50 \%$ risk and $85 \%$ $(n=23)$ of the carriers received an advice for regular surveillance (see Fig. 1). Ninety-one percent $(n=29)$ received their advice for regular surveillance via the clinical geneticist at the time of genetic counseling and testing. Three at risk individuals received indirect advice via the clinical geneticist (e.g., via their parents or brother/ sister who had undergone genetic counselling and testing and were proven to be carriers).

Those who received advice to undergo regular surveillance were significantly more likely to be carriers $(p=.01)$ and to be older (mean age $(s d)$ advice-versus no-advice- 
groep $43,7(14,2)$ versus $30,2(16,1) ; p=.01)$. No statistically significant differences were found between those who did and did not receive an advice for regular surveillance on the basis of gender, personal history of cancer or hospital.

Type of surveillance advice

Respondents reported having received very diverse advice on regular surveillance (Table 2). Of the total group, 78\% $(n=25)$ received advice for tailored screening, that is, organ-targeted surveillance based on family cancer history (e.g., colonoscopies at regular intervals if a relative has had colorectal cancer). Additionally, $72 \%(n=23)$ of the respondents reported having received advice to undergo an annual medical consultation, which includes an anamnesis and/or physical examination.

Twelve of the fourteen female respondents were 25 years of age or older (7 carriers and 5 at $50 \%$ risk). Only one of these women indicated that she had not been advised to undergo regular breast cancer surveillance. This was because she had undergone a bilateral mastectomy after having being diagnosed with two primary breast tumors. Five women were advised to undergo an annual mammography, 4 to undergo an annual breast MRI, and 2 women were advised to undergo both. Thus, in total, $64 \%$ $(n=7)$ of the female high risk family members aged 25 or older who received an advice to undergo surveillance for breast cancer were advised to undergo an annual mammography. Two of the 14 women were younger than

Table 2 Screening recommendations received by high risk LFS family members

\begin{tabular}{llll}
\hline Recommended screening & Carriers & $50 \%$ At risk & Total \\
\hline Males and females & $(n=23)$ & $(n=9)$ & $(n=32)$ \\
Annual medical consultation* & $18(78 \%)$ & $5(56 \%)$ & $23(72 \%)$ \\
Tailored screening $^{\mathrm{a}}$ & $19(83 \%)$ & $6(67 \%)$ & $25(78 \%)$ \\
Females $^{\mathrm{b}}$ & $(n=7)$ & $(n=7)$ & $(n=14)$ \\
Annual breast MRI $^{\mathrm{c}}$ & $3(43 \%)$ & $3(43 \%)$ & $6(43 \%)$ \\
Annual mammography $^{\mathrm{d}}$ & $5(71 \%)$ & $2(29 \%)$ & $7(50 \%)$
\end{tabular}

* Medical consultation includes a anamnesis and/or physical examination by a physician

${ }^{a}$ Tailored screening refers to organ-targeted surveillance based on family history, here including: colonoscopies $(n=9)$, endoscopic ultrasound pancreas $(n=4)$, MRI pancreas $(n=4)$, examination by a dermatologist $(n=14)$, ultrasound of kidneys $(n=10)$, urine analysis $(n=5)$, total blood count $(n=5)$

${ }^{\mathrm{b}}$ Of the 14 women, 12 were aged $\geq 25$ years or older ( 7 carriers and 5 at $50 \%$ risk), 2 were aged $<25$ years (both at $50 \%$ risk)

c All women who were advised to undergo a regular breast MRI were age 25 years or older

d All women who were advised to undergo a regular mammography were age 25 years or older
25 years of age and therefore no advice for breast cancer surveillance was given.

Adherence with the advice for regular surveillance

Of those who were advised to undergo regular surveillance for LFS, $78 \%(n=25)$ reported being fully compliant with that advice (see Fig. 1). Seven respondents (3 carriers and 4 at risk) indicated that they had not been (fully) adherent; they had not undergone (some of) the advised surveillance methods and/or not as frequently as advised. All woman who received advice about regular breast cancer surveillance reported being compliant $(n=11)$. Additionally, four individuals did not (fully) comply with the recommended medical consultations, and four with the tailored screening advice (colonoscopy $(n=2)$, ultrasound kidneys $(n=1)$, examination by a dermatologist $(n=1)$, endoscopic ultrasound pancreas $(n=1)$, total blood count $(n=2)$ ). Carriers tended to be more likely to be (fully) compliant with the advice for regular surveillance than those at $50 \%$ risk $(p=.08)$. No statistically significant differences were observed between compliers and noncompliers on the basis of gender, age, personal history of cancer or hospital.

\section{Reasons for non-compliance}

Of the seven individuals who reported not being (fully) compliant with the screening advice, two indicated that they did not believe that screening was particularly useful. Two other individuals indicated they did not undergo regular surveillance because they did not have any symptoms or complaints and therefore did not see any need for surveillance. One individual indicated avoiding regular surveillance because of fear of a tumor being detected, and two others stated that regular surveillance was too time consuming or difficult to plan.

Possible psychological gains of surveillance

\section{Perceived benefits of and barriers to screening}

The most prevalent perceived benefits of regular surveillance were early detection of cancer $(90 \%)$, a feeling of control $(84 \%)$, and a sense of security $(77 \%)$. The most important perceived barriers to regular surveillance were the possible negative effect on obtaining a mortgage, life insurance and/or health insurance (42\%), that it would cause unnecessary worry (36\%), and that it was impractical or inconvenient (e.g. in relation to work, childcare, etc.) (13\%) (see Table 3).

Those who indicated that they had followed the advice for regular surveillance were significantly more likely to 
Table 3 Perceived benefits \& barriers of screening for LFS $(n=31)$
* 1 case missing in analysis

a Significant difference $p \leq .05$

b Borderline significant $p \leq .10$

\begin{tabular}{|c|c|c|c|}
\hline & $\begin{array}{l}\text { Total } \\
(n=31)^{*} \\
\%\end{array}$ & $\begin{array}{l}\text { Adherent } \\
(n=25) \\
\%\end{array}$ & $\begin{array}{l}\text { Not (fully) } \\
\text { adherent } \\
(n=6) * \%\end{array}$ \\
\hline \multicolumn{4}{|l|}{ Benefits of screening } \\
\hline $\begin{array}{l}\text { Reduces the chance of cancer being detected } \\
\text { in an advanced stage }\end{array}$ & $90 \%$ & $96 \%{ }^{\mathrm{b}}$ & $67 \%^{\mathrm{b}}$ \\
\hline Reduces my fear of cancer & $61 \%$ & $72 \%^{\mathrm{a}}$ & $17 \%^{\mathrm{a}}$ \\
\hline Provides me with a feeling of control & $84 \%$ & $92 \%^{\mathrm{a}}$ & $50 \%^{\mathrm{a}}$ \\
\hline Gives me a sense of security & $77 \%$ & $84 \%$ & $50 \%$ \\
\hline Is a good way to detect cancer early & $90 \%$ & $92 \%$ & $83 \%$ \\
\hline Total scale score benefits (mean \pm SD) & $20.6 \pm 3.7$ & $21.3 \pm 3.4^{\mathrm{a}}$ & $17.7 \pm 3.6^{\mathrm{a}}$ \\
\hline \multicolumn{4}{|l|}{ Barriers of screening } \\
\hline $\begin{array}{l}\text { Can have a negative effect on my home mortgage } \\
\text { and/or life and health insurance }\end{array}$ & $42 \%$ & $44 \%$ & $33 \%$ \\
\hline Can cause unnecessary worry & $36 \%$ & $28 \%$ & $67 \%$ \\
\hline Causes inconvenience in my life & $13 \%$ & $8 \%$ & $33 \%$ \\
\hline $\begin{array}{l}\text { Screening is not important, if I am diagnosed with } \\
\text { cancer it will be too late }\end{array}$ & $3 \%$ & $4 \%$ & $0 \%$ \\
\hline Is painful & $0 \%$ & $0 \%$ & $0 \%$ \\
\hline Is uncomfortable/embarrassing & $10 \%$ & $8 \%$ & $17 \%$ \\
\hline Total scale score barriers (mean $\pm \mathrm{SD}$ ) & $12.8 \pm 3.2$ & $12.4 \pm 2.8$ & $14.0 \pm 4.6$ \\
\hline
\end{tabular}

report that regular surveillance reduced their fear of cancer ( $p=.02$ ) and that it provided them with a feeling of control $(p=.04)$. Those who were adherent were also more likely to indicate that regular surveillance reduces the chance of cancer being detected in an advanced stage ( $p=.09)$. Overall, those adherent with regular surveillance reported significantly more benefits from surveillance than those who were not (fully) adherent $(p=.03)$. No significant differences were found between the adherent and non-adherent groups with regard to the perceived barriers of regular surveillance (see Table 3 ).

\section{LFS-related distress and worries}

No statistically significant differences in psychological distress (IES) or worry (CWS) were found between those who received and adhered to advice for regular surveillance, those who received advise to undergo surveillance but did not do so (fully), and those who reported not having received advice for regular surveillance. For the total group, the mean score on the 'intrusion' subscale of the IES was $5.1(\mathrm{SD}=7.3)$ and on the CWS, $12.4(\mathrm{SD}=3.2)$. Nine individuals $(20 \%)$ scored above the cutoff of 9 on the IES, indicating clinically relevant levels of distress.

\section{Discussion}

To our knowledge, this study is the first to investigate which methods for regular surveillance, if any, are offered to high risk LFS family members, the extent to which such advice is followed, and the possible psychological burden or benefits associated with regular surveillance for health conditions associated with LFS. Although the actual medical benefits of surveillance for LFS are limited, the vast majority of LFS family members receive advice to undergo regular surveillance and report being adherent with the advice given.

Although the surveillance guidelines for LFS in the Netherlands solely include regular breast surveillance from age 20-25 years and an optional annual physical examination, this study shows that the specific screening advice given to high risk LFS family members in the Netherlands is more extensive and diverse than the guidelines. The large majority receive tailored screening advice based on family history. The national guidelines have recently been updated, and in these updated guidelines surveillance based on family history is included as optional [24]. This is in accordance with the US guidelines for regular surveillance published by the National Comprehensive Cancer Network [25].

Since regular surveillance for breast cancer is the only surveillance method considered to be effective, it is encouraging that all eligible high risk women aged 25 years or older received advice to undergo regular breast cancer surveillance, and adhere to this advice. It is notable that, despite the controversy about possible increased radiation sensitivity associated with p53 mutations [13, 26, 27], approximately two-thirds of these women were advised to undergo an annual mammography instead of a breast MRI. In addition, the large majority of these high risk individuals 
received tailored screening advice, based on their personal family history of cancer.

As hypothesized, individuals who received advice for regular surveillance and adhered to that advice reported greater benefits from regular surveillance, compared to those who were not (fully) adherent. The main perceived benefit of regular surveillance was the possible early detection of cancer. Additionally, regular surveillance provides a sense of control and security. Only limited barriers to surveillance were reported, and no differences regarding these barriers were found between those who were and were not (fully) compliant. However, although not significant, those who were not (fully) compliant with the advice received reported that regular surveillance may cause unnecessary worry, is inconvenient or can cause practical problems with finances or insurance.

Those who received and were compliant with advice for regular surveillance did not report significantly lower levels of LFS-related distress and worries than the non/undercompliers or those who reported not having being advised to undergo regular surveillance. At the same time, regular surveillance does not appear to result in any significant psychological burden for those who pursue it. Interestingly, as reported earlier, levels of distress and worry among LFS family members are, in general, relatively low and rather comparable to the more common forms of hereditary cancer [15].

Several limitations of this study should be noted. First, although we attempted to capture the entire LFS population in the Netherlands and the response rate was high (82\%), the sample size was relatively small. Larger international studies are needed to confirm our results. Second, the cross-sectional nature of the study only allows us to speak of statistical associations, rather than causal attributions. Relatedly, due to the small numbers and high compliance rate, it was not feasible to perform analyses to investigate which sociodemographic, clinical and psychosocial variables were associated with non-compliance at the multivariate level. Finally, it should be noted that information on advice received and compliance with that advice was based on self-report.

In summary, this study is the first to investigate the advice for regular surveillance given to high risk LFS family members, adherence with that advice, and perceived benefits of surveillance for LFS. Despite the fact that the medical gains of regular surveillance are limited, the vast majority of high risk family members are advised to undergo surveillance and are adherent with that advice. LFS family members have confidence in the ability of regular surveillance to detect cancer in an early stage, and thus this gives them a sense of control and security. Offering regular surveillance to high risk LFS family members may contribute to their psychological well-being.
Acknowledgments This study was financially supported by the Dutch Cancer Society (grant number NKI 2005-3209). We are grateful to all of the LFS family members who agreed to participate in this study.

Open Access This article is distributed under the terms of the Creative Commons Attribution Noncommercial License which permits any noncommercial use, distribution, and reproduction in any medium, provided the original author(s) and source are credited.

\section{References}

1. Malkin D, Li FP, Strong LC et al (1990) Germ line p53 mutations in a familial syndrome of breast cancer, sarcomas, and other neoplasms. Science 250:1233-1238

2. Chompret A, Brugieres L, Ronsin M et al (2000) P53 germline mutations in childhood cancers and cancer risk for carrier individuals. Br J Cancer 82:1932-1937

3. Hwang SJ, Lozano G, Amos CI et al (2003) Germline p53 mutations in a cohort with childhood sarcoma: sex differences in cancer risk. Am J Hum Genet 72:975-983

4. Hisada M, Garber JE, Fung CY et al (1998) Multiple primary cancers in families with Li-Fraumeni syndrome. J Natl Cancer Inst 90:606-611

5. Varley JM, Evans DG, Birch JM (1997) Li-Fraumeni syndrome-a molecular and clinical review. Br J Cancer 76:1-14

6. Jarvinen HJ, Mecklin JP, Sistonen P (1995) Screening reduces colorectal cancer rate in families with hereditary nonpolyposis colorectal cancer. Gastroenterology 108:1405-1411

7. Narod SA, Offit K (2005) Prevention and management of hereditary breast cancer. J Clin Oncol 23:1656-1663

8. Renkonen-Sinisalo L, Aarnio M, Mecklin JP et al (2000) Surveillance improves survival of colorectal cancer in patients with hereditary nonpolyposis colorectal cancer. Cancer Detect Prev 24:137-142

9. National Comprehensive Cancer Network (1999) NCCN practice guidelines: genetics/familial high risk cancer. Oncology 13:161-186

10. Schneider KA (2004) Li Fraumeni syndrome. Gene Rev (www. genetests.org)

11. Masciari S, Van den Abbeele AD, Diller LR et al (2008) F18-fluorodeoxyglucose-positron emission tomography/computed tomography screening in Li-Fraumeni syndrome. JAMA 299:1315-1319

12. Mitchel RE, Jackson JS, Carlisle SM (2004) Upper dose thresholds for radiation-induced adaptive response against cancer in high-dose-exposed, cancer-prone, radiation-sensitive $\operatorname{Trp} 53$ heterozygous mice. Radiat Res 162:20-30

13. Varley JM (2003) Germline TP53 mutations and Li-Fraumeni syndrome. Hum Mutat 21:313-320

14. Netherlands Foundation for the Detection of Hereditary Tumours, Dutch Society for Clinical Genetics: Hereditary Cancer: diagnostic and preventive guidelines (ed 3) (2005). Leiden, the Netherlands Foundation for the Detection of Hereditary Tumours, Dutch Society for Clinical Genetics, pp 14-16

15. Lammens C, Verhoef S, Aaronson N et al (2010) Genetic testing in Li-Fraumeni Syndrome: Uptake and psychosocial consequences. J Clin Oncol 28:3008-3014

16. Claes E, Denayer L, Evers-Kiebooms G et al (2004) Predictive testing for hereditary non-polyposis colorectal cancer: motivation, illness representations and short-term psychological impact. Patient Educ Couns 55:265-274

17. Champion VL (1984) Instrument development for health belief model constructs. ANS Adv Nurs Sci 6:73-85 
18. Kash KM, Holland JC, Halper MS et al (1992) Psychological distress and surveillance behaviors of women with a family history of breast cancer. J Natl Cancer Inst 84:24-30

19. Madalinska JB, van Beurden M, Bleiker EM et al (2007) Predictors of prophylactic bilateral salpingo-oophorectomy compared with gynecologic screening use in BRCA1/2 mutation carriers. J Clin Oncol 25:301-307

20. Brom D, Kleber RJ (1985) De schok verwerkingslijst [Impact of Event Scale]. Ned Tijdschr Psychol 40:164-168

21. Horowitz MJ (1986) Stress-response syndromes: a review of posttraumatic and adjustment disorders. Hosp Commun Psychiatr 37:241-249

22. Douma KF, Aaronson NK, Vasen HF et al (2010) Psychological distress and use of psychosocial support in familial adenomatous polyposis. Psycho-Oncol 19:289-298
23. Lerman C, Kash K, Stefanek M (1994) Younger women at increased risk for breast cancer: perceived risk, psychological well-being, and surveillance behavior. Monogr Natl Cancer Inst 16:171-176

24. Netherlands Foundation for the Detection of Hereditary Tumours (2010) Li-Fraumeni syndrome. Diagnostic and preventive guidelines, Hereditary cancer, pp 16-19

25. National Comprehensive Cancer Network (NCCN) (2010) The NCCN Clinical Practice Guidelines in Oncology ${ }^{\mathrm{TM}}$ Li-Fraumeni syndrome (Version 1.2010). NCCN. www.ncen.com

26. Eng C, Hampel H, de la Chapelle A (2001) Genetic testing for cancer predisposition. Annu Rev Med 52:371-400

27. Evans SC, Lozano G (1997) The Li-Fraumeni syndrome: an inherited susceptibility to cancer. Mol Med Today 3:390-395 